\title{
Effect of changes in the deuterium content of drinking water on the hydrogen isotope ratio of urinary steroids in the context of sports drug testing
}

\author{
Thomas Piper • Karoline Degenhardt • \\ Eugen Federherr • Andreas Thomas • \\ Mario Thevis • Martial Saugy
}

Received: 31 August 2012 /Revised: 11 October 2012 /Accepted: 16 October 2012 / Published online: 6 November 2012

(C) Springer-Verlag Berlin Heidelberg 2012

\begin{abstract}
The hydrogen isotope ratio (HIR) of body water and, therefore, of all endogenously synthesized compounds in humans, is mainly affected by the HIR of ingested drinking water. As a consequence, the entire organism and all of its synthesized substrates will reflect alterations in the isotope ratio of drinking water, which depends on the duration of exposure. To investigate the effect of this change on endogenous urinary steroids relevant to doping-control analysis the hydrogen isotope composition of potable water was suddenly enriched from -50 to $200 \%$ and maintained at this level for two weeks for two individuals. The steroids under investigation were $5 \beta$-pregnane- $3 \alpha, 20 \alpha$-diol, $5 \alpha$-androst- 16 -en- $3 \alpha$-ol, $3 \alpha$-hydroxy- $5 \alpha$-androstan-17-one (ANDRO), $3 \alpha$-hydroxy-
\end{abstract}

Published in the topical collection Isotope Ratio Measurements: New Developments and Applications with guest editors Klaus G. Heumann and Torsten C. Schmidt.

T. Piper $(\bowtie) \cdot$ K. Degenhardt $\cdot$ M. Saugy

Swiss Laboratory for Doping Analysis, University Center of Legal

Medicine, Geneva and Lausanne, Centre Hospitalier Universitaire

Vaudois and University Lausanne,

Ch. des Croisettes 22,

1066 Epalinges, Switzerland

e-mail: t.piper@biochem.dshs-koeln.de

E. Federherr

Elementar Analysensysteme $\mathrm{GmbH}$,

Donaustr. 7 ,

63452 Hanau, Germany

T. Piper $\cdot$ A. Thomas $\cdot$ M. Thevis

German Sport University Cologne, Center for Preventive Doping

Research-Institute of Biochemistry,

Am Sportpark Müngersdorf 6,

50933 Köln, Germany
$5 \beta$-androstan-17-one (ETIO), $5 \alpha$-androstane- $3 \alpha, 17 \beta$-diol, and $5 \beta$-androstane- $3 \alpha, 17 \beta$-diol (excreted as glucuronides) and ETIO, ANDRO and $3 \beta$-hydroxyandrost-5en-17-one (excreted as sulfates). The HIR of body water was estimated by determination of the HIR of total native urine, to trace the induced changes. The hydrogen in steroids is partly derived from the total amount of body water and cholesterol-enrichment could be calculated by use of these data. Although the sum of changes in the isotopic composition of body water was $150 \%$, shifts of approximately $30 \%$ were observed for urinary steroids. Parallel enrichment in their HIR was observed for most of the steroids, and none of the differences between the HIR of individual steroids was elevated beyond recently established thresholds. This finding is important to sports drug testing because it supports the intended use of this novel and complementary methodology even in cases where athletes have drunk water of different HIR, a plausible and, presumably, inevitable scenario while traveling.

Keywords Hydrogen isotope ratio - GC-TC-IRMS . Excretion study $\cdot$ Steroids $\cdot$ Enriched drinking water . Exchangeable hydrogen

\section{Introduction}

Recent investigation of hydrogen isotope ratios (HIR) of urinary steroids has demonstrated the potential and value of HIR in sports drug testing [1]. HIR are expressed as $\delta^{2} \mathrm{H}_{\mathrm{VSMO}}$ values against the international standard, 
Vienna Standard Mean Ocean Water (VSMOW), on the basis of Eq. (1):

$\delta^{2} H_{\text {VSMOW }}=\frac{\left(\frac{{ }^{2} H}{{ }^{1} H}\right)_{\text {SAMPLE }}}{\left(\frac{{ }^{2} H}{{ }^{1} H}\right)_{\text {VSMOW }}}-1$

where ${ }^{2} \mathrm{H} /{ }^{1} \mathrm{H}$ refers to the hydrogen isotope ratio of sample or standard [2].

Absolute $\delta^{2} \mathrm{H}_{\mathrm{VSMOW}}$ values obtained for urinary steroids vary over a $100 \%$ range, from -220 to $-320 \%$, for all investigated steroids and over approx. $50 \%$ for an individual steroid [1]. So, different endogenous steroids have significantly different HIR when excreted into urine, most probably because of isotopic fractionation or hydrogen exchange in the course of steroid metabolism or as a result of different isotopic composition of the precursor molecules at different endogenous production sites [3]. Consequently, absolute $\delta^{2} \mathrm{H}_{\text {VSMOw }}$ values are arguably not a promising option for detection of illicit steroid administration, so differences between steroids originating from discrete biological pathways were investigated. In accordance with carbon isotope ratio (CIR) determinations, analysis of distinct isotopic profiles is a valuable tool and endogenous reference compounds (ERC), for example pregnanediol (PD), and target compounds (TC), for example testosterone (TESTO), are considered and expressed as $\Delta^{2} \mathrm{H}$ values on the basis of Eq. (2):

$$
\Delta^{2} H=\delta^{2} H_{V S M O W}(E R C)-\delta^{2} H_{V S M O W}(T C)
$$

These $\Delta^{2} \mathrm{H}$ values established by means of reference population-based studies cover both the intra and interindividual variations and measurement uncertainties and can therefore be applied to sports drug testing in a straightforward manner [1, 4-7]. However, this is valid only as long as $\Delta^{2} \mathrm{H}$ values are stable within the individual athlete.

The objective of this study was to investigate the effect of a sudden change in the isotopic composition of drinking water on absolute $\delta^{2} \mathrm{H}_{\text {VSMOw }}$ and $\Delta^{2} \mathrm{H}$ values to ensure the validity of established thresholds. The activity of highprofile athletes necessitates a substantial travel around the world to attend competitions, and the HIR of potable water varies substantially, from +10 to $-150 \%$ [8-13]. So it can be assumed that the steady-state of hydrogen isotope distribution during steroid production within the body will be disturbed during traveling, which might result in significantly different $\Delta^{2} \mathrm{H}$ values. Such changes would challenge the diagnostic value of established thresholds and, therefore, of the entire approach.

The steroids under investigation to clarify this issue were: $5 \beta$-pregnane- $3 \alpha, 20 \alpha$-diol (PD), $5 \alpha$-androst- 16 -en- $3 \alpha$-ol (16EN), $5 \alpha$-androstane- $3 \alpha, 17 \beta$-diol ( $5 \mathrm{aDIOL}), 5 \beta$ androstane- $3 \alpha, 17 \beta$-diol (5bDIOL), $3 \alpha$-hydroxy- $5 \alpha$ - androstan-17-one (ANDRO), and $3 \alpha$-hydroxy- $5 \beta$-androstan-17-one (ETIO) excreted into urine as glucuronide conjugates. The steroid sulfates of ETIO, ANDRO, and $3 \beta$ hydroxy-androst-5-en-17-one (DHEA) were also studied.

The HIR of total native urine was also determined. By means of this information, changes in the isotopic composition of total body water can be monitored to examine the overall effects of the administered labeled water and to enable calculation of expected changes in body cholesterol. The HIR of the spiked drinking water was also measured. Moreover, hydrogen-exchange experiments were conducted to determine whether changes in HIR of urinary steroids can be attributed to exchangeable hydrogen atoms on the steroidal backbone. Finally, to ensure that metabolism of steroids is not affected by changes in the deuterium content of body water, urinary concentrations and CIR of excreted steroids were also monitored.

\section{Experimental}

\section{Chemicals and steroids}

Bakerbond SPE Octadecyl columns $(6 \mathrm{~mL}, 500 \mathrm{mg})$ were purchased from J.T. Baker (Deventer, Netherlands). Pyridine and acetic anhydride were from Sigma-Aldrich (Buchs, Switzerland) and $\beta$-glucuronidase from Escherichia coli was from Roche Diagnostics (Mannheim, Germany). tert-Butyl methyl ether (TBME) was obtained from Acros (Geel, Belgium), methanol, ethyl acetate, and sodium hydroxide from Merck (Darmstadt, Germany) and acetonitrile from Biosolve (Valkensward, Netherlands). All solvents and reagents were of analytical grade.

Steroid reference material 5aDIOL, 5bDIOL, 16EN, and $3 \beta$-hydroxy- $5 \alpha$-androstane (RSTD) were supplied by Steraloids (Newport, RI, USA). ANDRO, ETIO, PD, and DHEA were supplied by Sigma-Aldrich (Steinheim, Germany). Two secondary isotopic reference materials were purchased from A. Schimmelmann (Indiana University, IN, USA), namely the $n$-alkane mixture C3 (CAL1) and $5 \alpha$-androstane batch\#2 (CAL2) to calibrate the hydrogen tank gas (Carbagas, Gümlingen, Switzerland). Deuterium oxide $\left(99.9\right.$ atom $\left.\%{ }^{2} \mathrm{H}\right)$ was from Sigma-Aldrich. TESTO (17ß-hydroxy-androst-4-en-3-one) and ETIO-glucuronides were purchased from NMI (Sydney, Australia), ANDROglucuronide and DHEA-sulfate were from Sigma-Aldrich and ETIO and ANDRO-sulfates from Steraloids.

Administration study

\section{Participants}

Two male volunteers (aged 32 and 37 years, body weight 76 and $81 \mathrm{~kg}$ and height 175 and $180 \mathrm{~cm}$, respectively) 
participated in this study. Both subjects were healthy and declared not to use any prohibited substance or dietary supplement. During the study volunteers were asked not to change eating or drinking habits and to continue with their normal exercise ( 5 to $8 \mathrm{~h}$ per week). Both volunteers gave written consent and the study was approved by the ethics committee of the German Sport University.

\section{Administration procedure}

The hydrogen isotopic composition of drinking water can be expected to vary over a range of approximately $160 \%$ throughout the world $[9,11]$. To investigate the effects of sudden and pronounced changes it was decided to change the HIR by $250 \%$ for the sum of all water ingested during this study. For practical reasons, and to compensate for the significant contribution of food to the total amount of water ingested, it was decided to orally administer an aliquot $(0.25 \mathrm{~L})$ of highly enriched water in the morning and one in the evening with approx. $12 \mathrm{~h}$ in between. Supported by dietary records the total amount of water ingested by the individuals was estimated, to calculate the required HIR for the deuterium-enriched aliquots of drinking water [14].

At the beginning of the study the volunteers drank twice the amount of deuterium enriched water $(2 \times 0.5 \mathrm{~L})$ during the first two days of administration, to accelerate the changes to be induced. After two weeks of administration both volunteers returned to normal drinking water. To ensure homogenous labeling of drinking water a commercially available bottle for water dispensers containing $19 \mathrm{~L}$ was spiked with $5.5 \mathrm{~mL}^{2} \mathrm{H}_{2} \mathrm{O}$ and used as source of enriched water for the period of administration. This addition was calculated to change the $\delta^{2} \mathrm{H}$ value of the water by approx. $1500 \%$, which, assuming dilution by the factor of 6 , should result in an overall change of drinking water by $250 \%$. The dilution factor of 6 results from a supposed total water consumption of $3 \mathrm{~L}$ per day.

\section{Urine sampling}

Before the first administration, one blank morning urine was collected and then every morning urine and one spot urine in the evenings were sampled for the complete time of administration and for another two weeks after cessation. To monitor the complete return to basal value two morning urine samples were collected 6 and 7 weeks after the experiment had started. All specimens were stored frozen at $-20{ }^{\circ} \mathrm{C}$ until analysis.

For HIR determinations of endogenous steroids, all morning urine samples were investigated because of to the higher steroid content. Investigation of the HIR of total urine was performed for the first 25 days of the study.
Directly after changing the drinking water, both morning and evening urine was measured; otherwise only evening urine was determined. This procedure was chosen to minimize the number of samples under investigation but to trace changes properly wherever they were expected to be particularly pronounced.

\section{GC-MS measurements}

An aliquot of each specimen was prepared in accordance with routine doping-control sample-preparation procedures to determine the amount of endogenous steroids [15]. This enabled determination of the urine volume required for IRMS and ensured that urinary concentrations remained stable throughout the entire time period under investigation.

Because the steroid profile determined for each sample covers only glucuronidated steroids, the sulfates had to be quantified separately. An approach similar to one already published was chosen [7], using some simplifications, for example leaving out a sulfated internal standard, because mainly the volume required for reconstitution before isotope measurements was of interest and not the absolute amount of the steroid [1].

\section{Sample preparation}

Analytes have to be efficiently isolated and purified before gas chromatographic-thermal conversion-isotope ratio mass spectrometric (GC-TC-IRMS) analysis to avoid coelution of compounds and to maintain the ability to measure comparable amounts of differently concentrated urinary steroids. A detailed description of sample preparation to yield both glucuronidated and sulfated steroids including twofold high-performance liquid chromatography clean up has been published elsewhere and will, herein, only be described in brief $[1,6,7,16]$.

Urine $(10-30 \mathrm{~mL})$ was applied to a conditioned $\mathrm{C}_{18}$ solid-phase extraction cartridge. The column was washed with $2 \mathrm{~mL}$ water then eluted with methanol $(3 \times 1 \mathrm{~mL})$. The dried residue was dissolved in $1.5 \mathrm{~mL}$ of sodium phosphate buffer and extracted with $4 \mathrm{~mL}$ of TBME to separate unconjugated steroids; the aqueous residue was hydrolyzed with $\beta$-glucuronidase for $60 \mathrm{~min}$ at $50{ }^{\circ} \mathrm{C}$ and again extracted with $2 \times 4 \mathrm{~mL}$ TBME. The organic layer (containing formerly glucuronidated steroids) was evaporated to dryness, re-dissolved in $2 \times 100 \mu \mathrm{L}$ methanol, transferred to an HPLC autosampler vial, and evaporated.

The aqueous residue was acidified to $\mathrm{pH} 5$ with 100 $200 \mu \mathrm{L}$ glacial acid and applied to a conditioned $\mathrm{C}_{18}$ solid-phase extraction cartridge. After washing, the column was dried and stored in a vacuum desiccator overnight. Elution was performed with ethyl acetate- $\mathrm{MeOH}$ and after 
adding ethyl acetate-sulfuric acid the sample was incubated for $60 \mathrm{~min}$ at $50{ }^{\circ} \mathrm{C}$. Then $0.5 \mathrm{~mL}$ methanolic sodium hydroxide was added and the sample was evaporated to dryness, re-dissolved in water and extracted twice with $4 \mathrm{~mL}$ TBME to yield the formerly sulfated steroids. Half a milliliter of the organic layer was used for determination of steroid concentrations [7]; the remaining volume was evaporated, re-dissolved in $2 \times 100 \mu \mathrm{L}$ methanol, transferred to an HPLC autosampler vial, and evaporated.

HPLC fractionation was performed on an Agilent 1100 HPLC system with an XBridge Shield RP18 5- $\mathrm{mm}$ $(4.6 \mathrm{~mm} \times 250 \mathrm{~mm})$ column protected with an XBridge Shield RP18 5 - $\mu \mathrm{m}(4.6 \mathrm{~mm} \times 20 \mathrm{~mm})$ guard column purchased from Waters (Baden-Dättwil, Switzerland) [1].

\section{GC-TC-IRMS measurements}

\section{Instrumentation}

All samples were measured on an Agilent 6890 gas chromatograph coupled to a Delta ${ }^{\text {plus }}$ XL gas isotope-ratio mass spectrometer (ThermoElectron, Bremen, Germany) via a GC combustion interface (GCC III, ThermoElectron). Injections were performed in the splitless mode at $280{ }^{\circ} \mathrm{C}$ with injection volumes ranging from 2 to $4 \mu \mathrm{L}$ cyclohexane. The GC column was a J\&W Scientific DB-17MS (length $30 \mathrm{~m}$, i.d. $0.25 \mathrm{~mm}$, film thickness $0.25 \mu \mathrm{m}$ ) from Agilent. For steroid measurements, the initial oven temperature of $70{ }^{\circ} \mathrm{C}$ was maintained for $2 \mathrm{~min}$ and increased at $30^{\circ} \mathrm{min}^{-1}$ to $270{ }^{\circ} \mathrm{C}$, then at $2^{\circ} \mathrm{min}^{-1}$ to $290{ }^{\circ} \mathrm{C}$, then at $30^{\circ} \mathrm{min}^{-1}$ to $300^{\circ} \mathrm{C}$ and held for $1 \mathrm{~min}$. For CAL1 and CAL2, the initial oven temperature of $80{ }^{\circ} \mathrm{C}$ was maintained for 2 min and increased at $20^{\circ} \mathrm{min}^{-1}$ to $220^{\circ} \mathrm{C}$, then at $5^{\circ} \mathrm{min}^{-1}$ to $250{ }^{\circ} \mathrm{C}$, then at $20^{\circ} \mathrm{min}^{-1}$ to $300{ }^{\circ} \mathrm{C}$ and held for $2 \mathrm{~min}$. Carrier gas was purified $\mathrm{He}$ (purity grade 4.9) with a constant flow of 1.4 and $1.0 \mathrm{~mL} \mathrm{~min}^{-1}$, respectively. The thermal conversion furnace was operated at $1,450{ }^{\circ} \mathrm{C}$. Data were acquired by use of Isodat NT 2.0 software (ThermoElectron).

\section{Sequence alignment}

Each sequence of 24 to 36 samples was bracketed by a set of reference standard measurements. After determining the $\mathrm{H}_{3}{ }^{+}$-factor, a standard containing CAL2 and RSTD at $150 \mathrm{ng} \mathrm{mL}^{-1}$ was injected four times followed by threefold injection of a standard containing the relevant steroids of interest within the sequence plus RSTD at the same concentration. At the end of the sequence the same standards were injected three times each. The results obtained for the standards from before and after were compared ( $t$-test, $p<0.05$ ) and if no significant drift was detected, the sequence was accepted.
Blank urine

To each batch of samples $(n=6)$ blank urine was added to ensure valid measurements over time and to exclude any instrument drift as source of changes detected for urinary steroids. Frozen stored aliquots of the same pooled blank urine already used for method validation were processed [1]. This also enabled comparison of values obtained in this study with those from previous investigations.

\section{Calibration of tank gas}

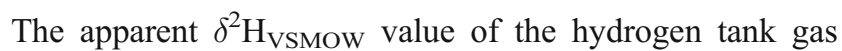
$(-337 \%)$ was calculated by using the mean value for all five $n$-alkanes contained in CAL1. The value obtained for the tank gas was confirmed by use of CAL2 and monitored constantly as CAL2 was injected with each batch of samples. With regard to the principle of identical treatment of standard and analytes [17], CAL2 was the only commercially available steroid reference material.

\section{Correction for the acetate moiety}

All determined values were corrected for the effect of the acetate moiety as described in literature [16, 18]. All $\delta^{2} \mathrm{H}_{\text {VSMOw }}$ values of urinary steroids reported within this article are for the underivatized steroid.

\section{EA-TC-IRMS measurements}

\section{Samples and sample preparation}

An in-house urine standard was collected in the morning from one male volunteer, filtered $(0.45 \mu \mathrm{m}$, cellulose nitrate), and transferred to 200 autosampler vials ( $1 \mathrm{~mL}$ each) with continuous homogenization with a magnetic stirrer. These standards and all urine samples were stored frozen at $-20{ }^{\circ} \mathrm{C}$ and placed in the refrigerator the day before measurement, for defrosting. Thirty minutes before transfer to auto-sampler vials they were homogenized and temperature equilibrated to room temperature. The urine samples and a set of five aqueous working standards (purchased from Elemental Microanalysis, Okehampton, UK) were not further pretreated. Each sample $(1 \mathrm{~mL})$ was placed in an autosampler vial.

\section{Sample analysis}

One microliter of each sample was injected, by use of a Vario liquid sampler, into the reaction tube of Vario Pyro cube (Elementar Analysensysteme, Hanau, Germany). The Pyro cube was run in HD-Cr reduction mode [19]. The hydrogen gas generated at $1080{ }^{\circ} \mathrm{C}$ in the $\mathrm{Cr}$ reactor was 
carried within the He stream $\left(200 \mathrm{mLmin}^{-1}\right)$ through an absorption tube $\left(\mathrm{NaOH}\right.$ and $\mathrm{P}_{4} \mathrm{O}_{10}$ on carrier) and thermal conductivity detector to an IsoPrime100 (Isoprime, Manchester, UK) IRMS. Reference gas pulses were introduced via the reference gas injector box (Isoprime). IRMS performance was monitored by use of regular stability and $\mathrm{H}_{3}{ }^{+}-$ correction tests using hydrogen reference gas. Reported $\delta^{2} \mathrm{H}_{\mathrm{VSMOW}}$ values were brought into agreement with accepted IAEA guidelines by using scale correction as described by Meier-Augenstein [20]. One difference was that four-point calibration with working standards was chosen because of the wide measurement range needed (from -100 to $+1700 \%$ ). The in-house urine standard was used to monitor and correct for possible drift over time. It was measured after each four samples. The contribution to uncertainty of the corrected sample $\delta^{2} \mathrm{H}_{\text {VSMOw }}$ values was taken into account via error propagation calculations.

Exchangeable hydrogen atoms bound to steroids

In contrast with the carbon atoms in the steroid backbone, with which no exchange can be expected, hydrogen atoms are prone to rapid equilibration with their environment. Changes in the isotopic composition of body water or urine might therefore affect the HIR of endogenous steroids directly without involving steroid metabolism pathways. Both direct hydrogen exchange and indirect incorporation of hydrogen from body water into cholesterol during biosynthesis were elucidated. To enable the differentiation of these effects, direct exchange of urinary steroids with hydrogen atoms from urine was investigated.

Therefore, one urine sample was split into five aliquots of $50 \mathrm{~mL}$. One was unaltered and the others were fortified with $2,4,8$, and $12 \mu \mathrm{L}$ deuterium oxide, respectively. This results in enrichment of urine water to approximately $+200,+450,+900$, and $+1500 \%$. The urine samples were left to equilibrate at room temperature for $1 \mathrm{~h}$ then processed as samples in accordance with the method. Steroids under investigation were ETIO, ANDRO and PD from the glucuronidated fraction and DHEA, ETIO and ANDRO from the sulfated fraction. Each sample was injected three times to minimize measurement uncertainty and to enable identification of even small differences introduced by the strong enrichment in deuterium of the urinary matrix.

Additionally, to check if the urinary matrix might have an effect, $5 \mathrm{~mL}$ water was spiked with steroid standards (glucuronidated TESTO, ETIO, and ANDRO and sulfated ETIO, ANDRO, and DHEA; $500 \mathrm{ng} \mathrm{mL}^{-1}$ each). Five of these steroid blanks were enriched as described above and processed together with the urine samples.

\section{CIR determinations}

All samples derived from the exchangeable hydrogen experiment and all aliquots containing PD, ETIO, and ANDRO from the drinking water change were also subjected to CIR determinations. Instrument and measurement details have been described in detail elsewhere [3]. For the urine enrichment the question arose whether these relatively high deuterium concentrations might affect subsequent CIR determinations in any way. For the drinking water switch, experiment CIR values were used to monitor any possible outstanding changes in metabolism, which should be mirrored by this isotope, also.

\section{Statistical analysis}

All statistical analysis was performed with IBM SPSS V20.0.0 software. Because changes in body water were monitored by EA-IRMS, changes in cholesterol could be calculated and compared with urinary HIR values of steroids. For one volunteer the changes in body water composition could be compared with the overall water administration to identify significant differences. For both total body water and total endogenous steroids single pool models were assumed; results from exponential curve fitting confirmed the assumption was sufficient for this investigation. Turnover of different steroid combinations within the body were investigated by use of Student's $t$-test for $a<0.05$, because HIR data can be assumed to be Gaussian distributed [1]. For effect of water and urine enrichment within the hydrogen exchange experiment was tested by applying linear models (LM) and probing for significant slopes $(a<0.05)$.

\section{Results and discussion}

\section{HIR of drinking water}

The HIR of the enriched 19-liter bottle of drinking water was determined to be $\delta^{2} \mathrm{H}_{\mathrm{VSMOW}}=1646 \pm 19 \%$; for the natural water $\delta^{2} \mathrm{H}_{\mathrm{VSMOW}}=-72 \pm 2.6 \%$. This magnitude of enrichment fit well with prior expectations and was sufficient to change the HIR of body water significantly.

HIR of body water

\section{EA-IRMS results}

Urine reflects the isotope ratio of body water for both hydrogen and oxygen isotopes and is regularly used as matrix for the doubly labeled water technique for estimation of energy expenditure [21]. Therefore, it is the ideal matrix 
to trace changes in body water composition. As depicted in Fig. 1, pronounced and very similar trends were observed for both subjects after changing the deuterium content of drinking water. The differences between the amount of enrichment for the subjects can most probably be ascribed to different amounts of overall water consumption during the administration period. Besides this, the slightly different isotopic composition of the additional water and food consumed might also have contributed.

During the wash out period from day 15 , completely parallel alterations in HIR values were observed for both subjects. Fitting of a one pool model using Eq. (3):

$y=B^{*} \ln (\mathrm{x})+\mathrm{C}$

resulted in equal values for the coefficient $B,-181.1 \pm 4.4$ and $-179.7 \pm 3.8$, and slightly different constants $C(581 \pm 13$ and $557 \pm 11$ ) because of different absolute enrichment at the time of completion of administration.

\section{Diet record fit}

Subject 2 completed a record of diet for the complete time of administration and by using this it was possible to estimate the real total water uptake per day. In total, taking into account both beverages and food, uptake was $3600 \pm$ $141 \mathrm{~mL}$ water per day. With this data the incremental increase in deuterium enrichment over time was calculated by simply adding the amount of enriched water balanced by total water intake. This resulted in the graph shown in Fig. 2. In comparison with the measured values this calculation slightly overestimates the deuterium content of body water. A possible explanation could be transfer of deuterium from total body water into biological compounds produced endogenously. The steep increase at the beginning is because

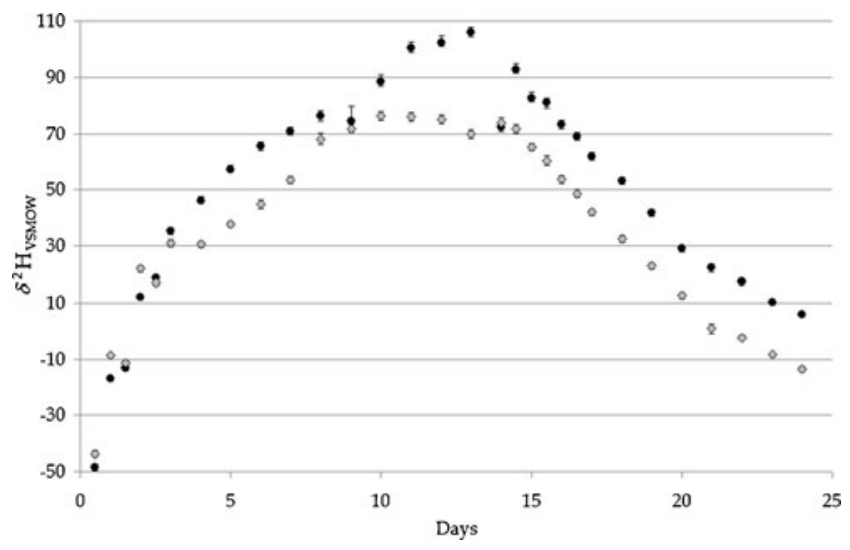

Fig. 1 Hydrogen isotopic composition of urine after changing the isotopic composition of drinking water on day one and returning to normal water on day 14. Black circles represent subject 1 and grey diamonds subject 2 . The error bars depict the measurement uncertainty of the EA-IRMS determinations

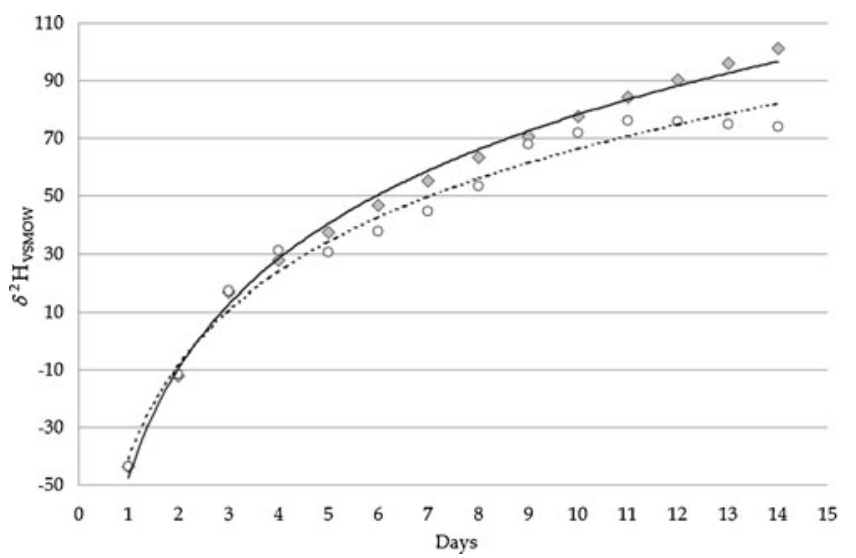

Fig. 2 Hydrogen isotopic composition of urine after changing the isotopic composition of drinking water on day one. Open circles represent the measured values for subject 2 and grey diamonds the incremental fitted values. The lines show the respective one pool model fit. Further information is given in the text

the amount of enriched water administered here was twice as much as during the remaining time.

\section{Theoretical changes in HIR of cholesterol}

Taking into account the above-mentioned changes in HIR of body water it is possible to estimate the induced changes in body cholesterol. The fractional rate of synthesis of new cholesterol entering the fast turnover total comprising that in red blood cells and the liver is known to be 6 to $8 \%$ [22-25]. Moreover, taking into account that only 22 out of 46 hydrogen atoms in cholesterol are derived from body water, the overall change in HIR of cholesterol can be estimated to increase slowly to a maximum of approx. 35 $\%$, as depicted in Fig. 3. This change should subsequently be incorporated throughout biosynthesis and metabolism of the steroid and become visible in the urinary metabolites of cholesterol.

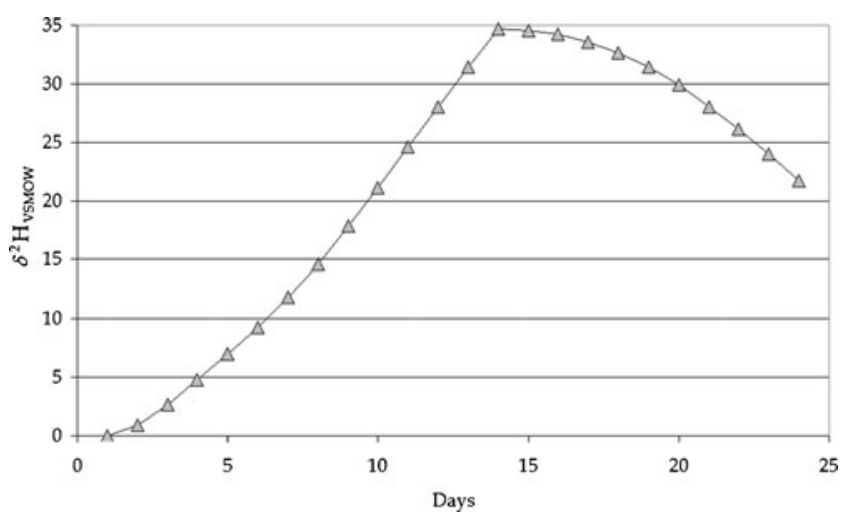

Fig. 3 Calculated change in cholesterol hydrogen isotopic composition induced by consumption of enriched drinking water from day 1 to day 14 


\section{Blank urine}

Results obtained for the sixfold preparation of the blank urine are listed in Table 1. During the period of 2 months in which measurements were conducted, no drift or significant change in HIR could be detected. This demonstrates the repeatability of the whole method over time and excludes any measurement uncertainty or drift as being responsible for the detected changes in HIR presented later. Besides this, comparing the values obtained during this study with already published values gathered during reference population determinations, a stable offset of $5 \%$ for all steroids is visible [1]. This might be a result of changes in HIR during long-term storage of the urine, but the data available were insufficient to confirm this effect. Further studies might be necessary here. Of importance to this study was the stability over a shorter time, which is proved by the data.

\section{Steroid concentrations}

Urinary concentrations were determined to ensure proper reconstitution of each analyte before IRMS and to trace possible changes in steroid metabolism induced by deuterium enrichment. Throughout the study, concentrations within each subject remained stable. As expected, morning urine always had a higher steroid content and slightly less variability than urine collected in the evening. No significant changes over time were detectable and coefficients of variation were in comparable ranges with those in other studies, i.e. between $20 \%$ and $30 \%[15,26,27]$. As for the reference population, no significant correlation $(p<0.05)$ was detectable between steroid concentration and the corresponding HIR.

\section{Changes in HIR of urinary steroids}

All the results obtained are summarized in Fig. 4. Similar parallel, overall changes to the extent expected from our

\begin{tabular}{|c|c|c|c|}
\hline \multirow{7}{*}{$\begin{array}{l}\text { processed with each } \\
\text { batch of samples, } n=6 \text {, } \\
\text { over a time period of } \\
2 \text { months }\end{array}$} & Steroid & Mean & $\mathrm{SD}$ \\
\hline & $\operatorname{RSTD}^{\mathrm{a}}$ & -323.9 & 7.1 \\
\hline & ETIO & -293.9 & 4.3 \\
\hline & ANDRO & -279.5 & 4.3 \\
\hline & $5 \mathrm{aDIOL}$ & -273.9 & 5.7 \\
\hline & $5 \mathrm{bDIOL}$ & -290.2 & 3.7 \\
\hline & PD & -251.3 & 5.3 \\
\hline \multirow{2}{*}{$\begin{array}{l}\text { avalue for all } 54 \\
\text { measurements }\end{array}$} & $16 \mathrm{EN}$ & -268.1 & 5.9 \\
\hline & ETIO_Sulf & -286.7 & 4.2 \\
\hline \multirow{2}{*}{$\begin{array}{l}\text { All values in } \delta^{2} \mathrm{H}_{\text {VSMOW }} \\
\text { [\%o] are for underivat- } \\
\text { ized steroid }\end{array}$} & ANDRO_Sulf & -273.8 & 4.5 \\
\hline & DHEA_Sulf & -281.1 & 1.5 \\
\hline
\end{tabular}

calculations for changes in the HIR of cholesterol (vide supra) were observed for all the steroids under investigation. The mean maximum change for glucuronidated steroids (PD, 16EN, ANDRO, ETIO, 5aDIOL, and 5bDIOL) was comparable for both subjects ( $36 \pm 4$ and $37 \pm 3 \%$ ). The response was less distinctive for the sulfated steroids excreted (28 \pm 4 and $25 \pm 4 \%$ for subjects 1 and 2, respectively).

Regarding the ERCs, the subjects responded slightly differently to the water change. Whereas for subject 2 similarly modified HIR values were obtained for both $16 \mathrm{EN}$ and $\mathrm{PD}$, for subject $116 \mathrm{EN}$ seemed to be affected more than PD. This is also reflected by the coefficients for the applied model calculations listed in Table 2. Subject 1 excreted less $16 \mathrm{EN}\left(335 \pm 142 \mathrm{ngmL}^{-1}\right)$, which might be indicative of a smaller amount of $16 \mathrm{EN}$ inside the body of this volunteer, and might explain the faster change of values after the induced changes in body water composition. In contrast, this subject excreted large amounts of PD $(1075 \pm 437 \mathrm{ng}$ $\mathrm{mL}^{-1}$ ) and again, if we assume that this reflects a large amount of PD within the body, this would explain the relatively slow response assuming that the turnover for all steroids follows similar kinetics. For subject 2, both steroids were excreted in similar amounts with slightly higher concentrations found for $16 \mathrm{EN}$ (300 and $500 \mathrm{ng} \mathrm{mL}^{-1}$ respectively) and changes in HIR occur at similar rates. Only the variability of $16 \mathrm{EN}$ was found to be larger here than for PD.

Among the glucuronidated TCs, a parallel shift in HIR values was observed for all steroids; for the sulfated TCs some differences can be observed. Overall there is a tendency for a less pronounced response, although this is not significant in subject 1 . For subject 2 it is significant for both ETIO and, especially, DHEA (Table 2). Here the smallest change in HIR during the administration period, only 22 $\%$, was determined. This again corresponds to large amounts of DHEA-sulfate excreted in the urine by this subject. In addition, subject 2 excreted, overall, more sulfated steroids than subject 1 , which might explain the less pronounced response by these conjugates. For subject 1 measurements of DHEA sulfate were not possible, because of the small amount in the urine.

With the data available it is, of course, not possible to unambiguously identify the source of the slightly different changes in HIR, but considering also urinary concentrations, it seems obvious that less distinct changes are likely to be observed for steroids excreted in urine to a greater extent. These differences might affect established reference based values for $\Delta^{2} \mathrm{H}$ values.

$\Delta^{2} \mathrm{H}$ values and reference limits

If an ERC changed more quickly (or more slowly) than the TCs, as found for subject 1's 16EN and PD, this might cause a problem in the context of doping control analysis. In this 
Fig. 4 Hydrogen isotopic composition of endogenous urinary steroids over time. (a) ERCs, open diamonds represent $\mathrm{PD}$, grey circles $16 \mathrm{EN}$, the lines represent the moving average. (b) TCs excreted glucuronidated, open triangles represent 5aDIOL, black triangles ANDRO, grey squares ETIO, open squares $5 \mathrm{bDIOL}$, and the lines, as typical examples, represent the moving average for ETIO. (c) TCs excreted sulfated, open circles represent ANDRO, black crosses ETIO, black circles DHEA, and lines again represent the moving average for ETIO. All values are for underivatized steroids
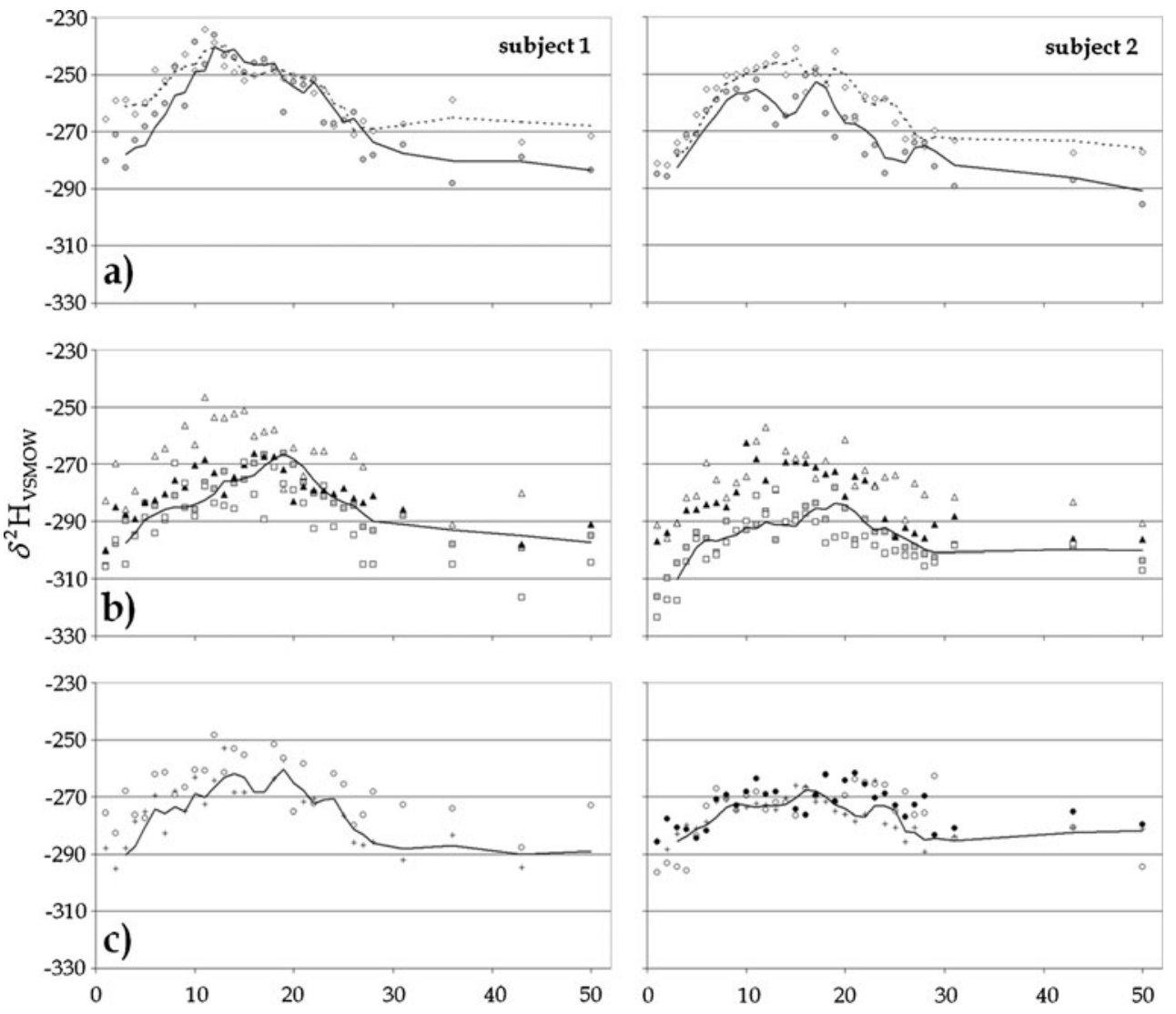

case the established reference-based thresholds for distinguishing between an endogenous or exogenous source of steroid might lose their informative value, or at least suffer the loss of some statistical significance. So all $\Delta^{2} \mathrm{H}$ values were carefully investigated throughout the study and all were found to be within established limits not resulting in any false positive (i.e. elevated beyond the threshold) sample. The mean values and corresponding standard deviations are listed in Table 3. As expected, the scatter of the data is between that of the blank urine processed within the study (Table 1) and that for the reference population [1].
Nevertheless, some trends are visible as depicted in Fig. 5. In particular for subject 1 it is obvious that the faster change of $16 \mathrm{EN}$ at the beginning of the study results in elevated $\Delta^{2} \mathrm{H}$ values for pairs of ERC and TC built with this steroid. The mean average for 16EN-ETIO is $17 \%$ and that for $16 \mathrm{EN}-\mathrm{ANDRO}$ is approximately $14 \%$, and those values increased to maxima of $47.5 \%$ and $37.3 \%$, respectively. Both values are close to the established reference-based values of $51.6 \%$ and $38.6 \%$ [1]. In contrast, for PD the reverse trend is apparent, and $\Delta^{2} \mathrm{H}$ values decrease during the study from 30 to $40 \%$ to 10 to $20 \%$ for PD-ETIO and
Table 2 Estimated coefficients and corresponding standard errors obtained by fitting of a one-pool model (Eq. 3) to the HIR data for urinary steroids

*Significantly different, $p<0.05$

\begin{tabular}{|c|c|c|c|c|}
\hline \multirow[t]{2}{*}{ Steroid } & \multicolumn{2}{|l|}{ Subject 1} & \multicolumn{2}{|l|}{ Subject 2} \\
\hline & Coefficient B & Std. error & Coefficient B & Std. error \\
\hline ETIO & 10.9 & 1.2 & 10.9 & 1.0 \\
\hline ANDRO & 9.6 & 1.5 & 11.4 & 1.8 \\
\hline $5 \mathrm{aDIOL}$ & 13.3 & 2.3 & 12.1 & 2.2 \\
\hline $5 \mathrm{bDIOL}$ & 10.9 & 2.0 & 15.0 & 1.6 \\
\hline PD & 8.5 & 2.1 & 15.5 & 1.9 \\
\hline $16 \mathrm{EN}$ & $17.3^{*}$ & 3.1 & 13.6 & 1.5 \\
\hline ETIO_Sulf & 11.5 & 2.4 & $7.7^{*}$ & 1.0 \\
\hline ANDRO_Sulf & 9.7 & 1.9 & 12.0 & 2.2 \\
\hline DHEA_Sulf & nd & nd & $5.7 *$ & 1.8 \\
\hline
\end{tabular}


Table 3 Mean values and standard deviations (SD) for all pairs of steroids under investigation over the complete trial

\begin{tabular}{lccccc}
\hline \multirow{2}{*}{ Pair of steroids } & \multicolumn{2}{c}{ Subject 1} & & & \multicolumn{2}{c}{ Subject 2} & \\
\cline { 2 - 3 } \cline { 5 - 6 } & Mean & SD & & Mean & SD \\
\hline PD-ETIO & 28.3 & 8.6 & & 34.4 & 7.6 \\
PD-ANDRO & 24.6 & 7.2 & & 21.9 & 6.9 \\
PD-5aDIOL & 12.8 & 8.5 & & 14.9 & 7.1 \\
PD-5bDIOL & 34.3 & 8.3 & & 37.7 & 7.5 \\
PD-ETIO_Sulf & 20.9 & 8.0 & & 16.3 & 8.2 \\
PD-ANDRO_Sulf & 11.3 & 8.2 & & 14.3 & 10.4 \\
PD-DHEA_Sulf & nd & nd & & 12.1 & 10.4 \\
16EN-ETIO & 22.1 & 9.7 & & 25.0 & 9.7 \\
16EN-ANDRO & 18.5 & 9.3 & & 12.1 & 8.2 \\
16EN-5aDIOL & 7.0 & 8.1 & & 7.0 & 9.8 \\
16EN-5bDIOL & 28.5 & 10.1 & & 28.3 & 9.8 \\
16EN-ETIO_Sulf & 14.5 & 9.2 & & 6.9 & 9.2 \\
16EN-ANDRO_Sulf & 4.8 & 11.1 & & 4.9 & 11.7 \\
16EN-DHEA_Sulf & nd & nd & 2.9 & 11.2 \\
\hline
\end{tabular}

For each subject $n=31$ individual measurements were performed

PD-ANDRO, which would benefit the athlete because it would hamper detection of exogenous steroids. For subject 2 no similar trend is visible, here the values scatter around the mean; only for $16 \mathrm{EN}$ as ERC is a slight trend of approx. $10 \%$ apparent over the complete period of seven weeks.

To summarize these findings, the change of drinking water can affect $\Delta^{2} \mathrm{H}$ values relevant to doping control analysis. But, within this study, no value was detected above the established thresholds, despite the fact that the change in HIR of drinking water was twice that which can be expected under normal conditions, i.e. during traveling. Regarding the reported values for HIR in water, it is impossible to mimic the change that was induced within this study simply by chance. Even the worst possible change of drinking water (which would result in a change of $160 \%[9,11]$ ) would be buffered by all other sources of water administered (i.e. food and other beverages). To induce the strong change used in this study, water with a difference in HIR of more than $1600 \%$ was administered, resulting in a change of overall administered water of $250 \%$. So it would be extremely unlikely to find a false positive case solely as a result of variations in drinking water, especially so because it cannot be expected that differences in $\Delta^{2} \mathrm{H}$ values will be equally affected for all ERCs, as demonstrated for PD and $16 \mathrm{EN}$ in subject 1 .

\section{Exchangeable hydrogen atoms}

Apart from some early work on exchangeable hydrogen atoms in cholesterol [28], not much is found in the literature on this topic, especially investigations on this exchange under "normal" conditions, i.e. conditions usually found within urine. Although fast-equilibrating hydrogen atoms in $\mathrm{OH}$ groups are eliminated during derivatization, it is not known whether:

1. vicinal hydrogen atoms exchange with the surrounding water directly;

2. whether they exchange during the chemical reaction of derivatization; or

3. whether these atoms can be replaced during the cleavage of the glucuronide or sulfate moiety.

In acetone, for example, hydrogen atoms exchange slowly under these "normal" conditions whereas those in acetic
Fig. 5 Typical $\Delta^{2} \mathrm{H}$ values over time depicted for both subjects, for (a) PD-ETIO (grey squares) and PD-ANDRO (open squares) and (b) 16ENETIO (grey diamonds) and 16EN-ANDRO (open diamonds)
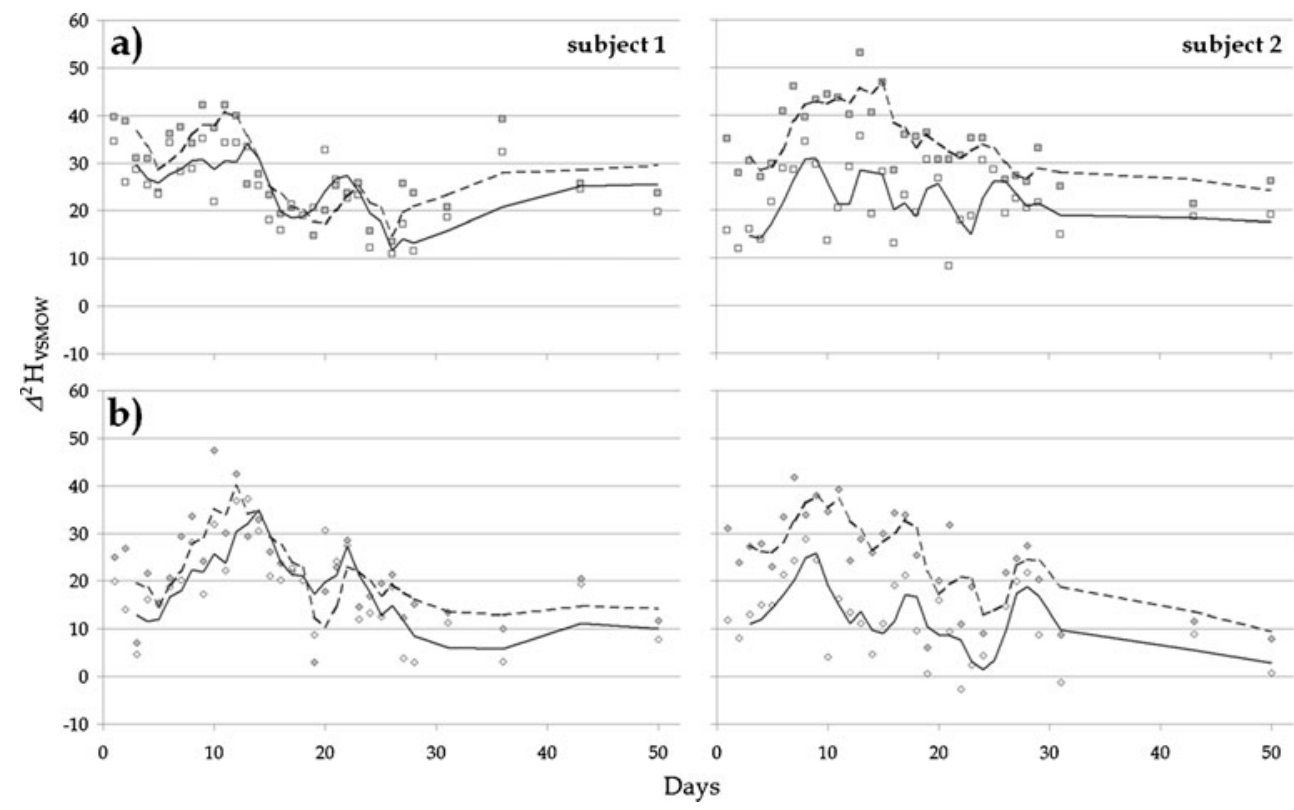
acid do not $[28,29]$. To ensure that the changes detected in HIR are not because of these direct effects of urine on excreted steroids, we substantially changed the HIR of urine (and water fortified with steroid conjugates) and performed complete sample preparation with these aliquots to identify any exchange of hydrogen atoms.

In accordance with the early findings, no change in HIR was detected for any of the steroids under investigation, although it must be mentioned that the first investigation in 1937 on this topic sought percent differences for deuterium. Our results are listed in Table 4.

For neither urinary steroids nor the standards added to water could any effect of the surrounding medium be confirmed. Each steroid at one level of enrichment was tested against all other levels of enrichment ( $t$-test, $a<0.05)$ and no significant difference was found. Additionally, all steroid HIR obtained were plotted against the magnitude of enrichment but no linear relationship with a slope significantly different from 0 (LM, $a<0.05$ ) could be detected. The only visible abnormality was the relatively high standard deviation for ANDRO-glucuronide added to water. This might be because of known difficulties in the cleavage of this conjugate during enzymatic hydrolysis [15, 30], but no further investigations were conducted because this question was beyond the focus of this study.

In conclusion, it can be stated that variations in the deuterium content of the surrounding medium do not have any effect on the HIR of the measured steroids after performing the complete sample preparation. So, during

Table 4 Mean values and standard deviations (SD) for all the steroids under investigation in the hydrogen-exchange experiment

\begin{tabular}{llrr}
\hline Matrix & Steroid & Mean & SD \\
\hline Urine & RSTD* & -326.4 & 4.4 \\
& ETIO & -280.8 & 2.9 \\
& ANDRO & -272.9 & 1.4 \\
& PD & -249.2 & 3.0 \\
& ETIO_Sulf & -282.1 & 2.6 \\
Water+standards & ANDRO_Sulf & -272.6 & 1.3 \\
& DHEA_Sulf & -272.4 & 2.7 \\
& RSTD* & -327.7 & 6.6 \\
& ETIO & -207.3 & 3.5 \\
& ANDRO & -308.5 & 10.6 \\
& TESTO & -228.9 & 3.9 \\
& ETIO_Sulf & -184.3 & 4.5 \\
& ANDRO_Sulf & -133.9 & 5.6 \\
& DHEA_Sulf & -277.9 & 4.5 \\
\hline
\end{tabular}

Listed are the mean values and standard deviations for $n=15(* 105)$ injections

All values in $\delta^{2} \mathrm{H}_{\mathrm{VSMOW}}[\%$ ] for underivatized steroid. Further information is given in the text de-conjugation no hydrogen atoms from the medium were incorporated into the steroid and derivatization completely substitutes any exchangeable hydrogen atom.

\section{CIR of urinary steroids}

No effect could be detected, either for the excretion study or for the exchange experiment. For the samples investigated from each subject $(n=31)$ standard deviations for $\delta^{13} \mathrm{C}$ values range from 0.2 to $0.4 \%$; for $\Delta^{13} \mathrm{C}$ values they ranged from 0.4 to $0.5 \%$, which is in perfect agreement with the technical capabilities of the method [3,6]. No significant trends over time were apparent. As expected, similar low standard deviations ( 0.2 to $0.4 \%$ ) were obtained for CIR for the deuterium-enriched urine samples.

\section{Conclusion}

We carefully investigated whether a sudden change in the deuterium content of an athlete's drinking water induced by traveling, for example, can affect HIR measurements of urinary steroids in the context of doping control analysis. For our two volunteers, enrichment of overall water intake by $250 \%$ resulted in significant changes of HIR for all the steroids investigated. Because these changes occur predominantly in parallel, effects on $\Delta^{2} \mathrm{H}$ values were detected but no elevation of any of these differences beyond established thresholds could be confirmed. Taking into account that the induced changes here were more than double those that might be expected for real-life water changes for an athlete, we conclude that false positive findings above a defined threshold would be extremely unlikely, especially if different ERCs are considered.

Because no direct hydrogen exchange could be detected for any of the steroids investigated, this possible source of interference can be excluded. Changes apparent for HIR of excreted steroid metabolites should, therefore, only be because of changes in the overall precursor of endogenous steroids, cholesterol, which is de-novo synthesized by the body. Additional effects on HIR of steroid metabolites during metabolism, for example introduction of hydrogen atoms during the reduction of double bonds in TESTO or DHEA by enzymes using hydrogen derived from NADPH, cannot be excluded on the basis of the data available, but were not further identifiable with the chosen experiment. The differences found in turnover times of different steroids are most probably because of the different amounts present but, again, further studies would be necessary for clarification.

Changes in the deuterium content of body water seem not to affect steroid metabolism in any way, because both the 
urinary concentrations and CIR values remained stable throughout this study.

One possible disadvantage of using HIR in sports drug testing, rapid changes of the deuterium content of drinking water, can be excluded as having a significant effect on this new method.

Acknowledgments This project was partly funded by the Partnership for Clean Competition (PCC), the Federal Ministry of the Interior of the Federal Republic of Germany and the Manfred-Donike-Institute for Doping Analysis (MDI), Cologne. The authors would like to thank Dr Karsten Köhler for his assistance in interpreting the dietary records.

\section{References}

1. Piper T, Thomas A, Thevis M, Saugy M (2012) Drug Test Anal 4:717-727

2. Coplen TB (2011) Rapid Commun Mass Spectrom 25:2538-2560

3. Piper T, Baume N, Strahm E, Emery C, Saugy M (2012) Steroids 77:644-654

4. Solberg HE (1993) J Int Fed Clin Chem 5:162-165

5. Flenker U, Güntner U, Schänzer W (2008) Steroids 73:408-416

6. Piper T, Mareck U, Geyer H, Flenker U, Thevis M, Platen P, Schänzer W (2008) Rapid Commun Mass Spectrom 22:2161-2175

7. Piper T, Opfermann G, Thevis M, Schänzer W (2010) Rapid Commun Mass Spectrom 24:3171-3181

8. Friedman I (1953) Geochim Cosmochim Acta 4:89-103

9. Bowen GJ, Winter DA, Spero HJ, Zierenberg RA, Reeder MD, Cerling TE, Ehleringer JR (2005) Rapid Commun Mass Spectrom 19:3442-3450

10. Brencic M, Vreca P (2006) Rapid Commun Mass Spectrom 20:3205-3212

11. Bowen GJ, Ehleringer JR, Chesson LA, Stange E, Cerling TE (2007) Water Resour Res 43:W03419
12. Kim GE, Ryu JS, Shin WJ, Bong YS, Lee KS, Choi MS (2012) Rapid Commun Mass Spectrom 26:195-204

13. Data available at: http://wateriso.eas.purdue.edu/waterisotopes/ index.html (accessed 27.07.2012)

14. Koehler K, Braun H, De Marees M, Fusch G, Fusch C, Mester J, Schänzer W (2010) J Sports Sci 13:1435-1449

15. Mareck U, Geyer H, Opfermann G, Thevis M, Schänzer W (2008) J Mass Spectrom 43:877-891

16. Piper T, Thevis M, Flenker U, Schänzer W (2009) Rapid Commun Mass Spectrom 23:1917-1926

17. Werner RA, Brand WA (2001) Rapid Commun Mass Spectrom 15:501-519

18. Docherty G, Jones V, Evershed P (2001) Rapid Commun Mass Spectrom 15:730-738

19. Morrison J, Brockwell T, Merren T, Fourel F, Phillips AM (2001) Anal Chem 73:3570-3575

20. Meier-Augenstein W (2010) In: Meier-Augenstein W (ed) Stable isotope forensics: an introduction to the forensic application of stable isotope analysis. Wiley, Oxford, pp 85-90

21. Schoeller DA, Colligan AS, Shriver T, Avak H, Bartok-Olson C (2000) J Mass Spectrom 35:1128-1132

22. London IM, Rittenberg D (1950) J Biol Chem 184:687-691

23. Goodman DS, Noble RP (1968) J Clin Invest 47:231-241

24. Goodman DWS, Smith FR, Seplowitz AH, Ramakrishnan R, Dell RB (1980) J Lipid Res 21:699-713

25. Neese RA, Faix D, Kletke C, Wu K, Wang AC, Shackleton CHL, Hellerstein MK (1993) Am J Physiol Endocrinol Metab 264: E136-E147

26. Zimmerman J (1986) Untersuchungen zum Nachweis von exogenen Gaben von Testosteron. Thesis, Deutsche Sporthochschule Köln, Cologne

27. Rauth S (1994) Referenzbereiche von urinären Steroidkonzentrationen und Steroidquotienten. Thesis, Deutsche Sporthochschule Köln, Cologne

28. Schoenheimer R, Rittenberg D (1937) J Biol Chem 121:235-253

29. Halford JO, Anderson LC, Bates JR (1934) J Am Chem Soc 56:491-492

30. Vestergaard P (1978) Acta Endocrinol Suppl 217:96-120 